\title{
Anticoagulant Induced Conus Hematomyelia with Subarachnoid Extension.
}

\author{
Kalyan Bommakanti ${ }^{1}$, Pavan Kumar Pelluru ${ }^{2}$, Barada Prasad Sahu ${ }^{3}$, \\ Chandu Lingolu ${ }^{4}$ \\ Department Of Neurosurgery, Nizam's Institute Of Medical Sciences, Hyderabad, India
}

\begin{abstract}
Anticoagulant induced hematomyelias are rare and a few cases are reported in the literature. They are commonly located in the thoracic cord and are assosciated with a poor prognosis even with aggressive medical and surgical treatment. Only case of anticoagulant induced conus hematomyelia is reported. Here with, we are reporting a case of conus hematomyelia who had an excellent neurological recovery with prompt reversal of anticoagulation and early surgical evacuation of hematoma.
\end{abstract}

Keywords: Hematomyelia; intramedullary; hematoma; anticoagulants; warfarin.

\section{Introduction}

Hematomyelia refers to intramedullary bleeding in to the spinal cord. ${ }^{5}$ Anticoagulant induced spinal hematomas are well reported in the literature. They are commonly seen in the epidural space. Few cases of anticoagulant induced spinal subdural hematomas have been reported. ${ }^{4}$ Anticoagulant induced hematomyelia's are very rare and only one case of anticoagulant induced conus hematomyelia is reported in the literature. ${ }^{3,7}$ Here in we report a case of conus hematomyelia with subarachnoid extension who had excellent neurological recovery after surgical evacuation.

\section{Case Report}

A 55 year old female patient with severe mitral stenosis underwent mitral valvotomy 2 years ago and was on warfarin ( $5 \mathrm{mg}$ once daily). She was on irregular follow up and dosage of warfarin was not monitored. She presented with complains of breathlessness to the department of cardiology. She was evaluated appropriately by the cardiologist, found to have atrial fibrillation and was being managed with diuretics, oxygen supplementation and digoxin. There were no episodes of hypertension during her stay in hospital. There were no signs of bleeding like epistaxis or bruises but the international normalized ratio (INR) was 7. So, warfarin was stopped. On the second day, the patient developed sudden low backache, flaccid weakness of both lower limbs (Medical research council grade 1/5), decreased sensations in both the lower limbs till the groin region bilaterally and bladder retention. Magnetic resonance (MR) imaging of thoracolumbar spine showed evidence of intramedullary bleed at conus (L1 vertebral level) and hematoma filling the thecal sac up to sacrum. The hematoma was isointense to hypointense to cord parenchyma on T1W images and hyperintense to cord parenchyma on T2 weighted images. There was cord edema extending vertically up to D-10 vertebral level (Fig 1). The coagulation parameters were immediately corrected with fresh frozen plasma infusions, Vitamin $\mathrm{K}$ and the patient was taken up for surgery. L1 and L2 laminectomy was done. Dura and arachnoid were opened. The subarachnoid space was filled with blood clot which was evacuated. Conus was discoloured brownish-red due to hematoma. A midline myelotomy revealed a dark, liquefied haematoma (Fig 2). This was evacuated and the cord surrounding this collapsed and began to pulsate normally. Hemostasis was secured and dura was closed. On $2^{\text {nd }}$ post operative day the power in lower limbs improved to medical research council grade $3 / 5$ and she was on foley's catheter. At three months follow up, the patient was ambulatory with minimal support and was off Foleys catheter.

\section{Discussion}

Spinal hematomas are well known to be assosciated with the use of anticoagulants. Spinal hematomas can be epidural, subdural or intramedullary. Epidural spinal hematomas are common but subdural and intramedullary hematomas are rare. There are few case reports of anticoagulant induced subdural hematomas but intramedullary hematomas (hematomyelia's) are very rare. ${ }^{2,4,61,3-5,7,8}$ Anticoagulant induced intramedullary hematoma in the conus region is very rare and, as per our knowledge, only one case of anticoagulant induced conus hematomyelia has been reported in the literature. ${ }^{7}$

Intramedullary hematomas can be caused by a variety of reasons. Spinal arteriovenous malformations and cavernomas are the most common causes of non-traumatic intramedullary hematomas. Primary spinal cord tumors, spinal cord metastases, anticoagulants, bleeding disorders, spinal cord irradiation are other causes of 
intramedullary hematomas. ${ }^{5}$. Giuseppe et al have found 9 patients with warfarin induced hematomyelias reported in the literature. They were most common in the thoracic spine followed by cervical spine. Only one patient had hematoma in the conus region. Majority of them presented with localized or radicular pain, rapidly progressive paraplegia/ quadripledia and urinary retention. ${ }^{3}$. Our patient had intramedullary hematoma in the conus region with subarachnoid extension and had presented with severe low back ache, paraparesis and bladder retention.

With increasing use of potent and long term anticoagulants, the incidence of bleeding in unusual sites may increase. So hematomyelia should be considered in all patients on anticoagulants who present with paraparesis/quadriparesis. As the number of cases reported in the literature are few, guidelines cannot be laid down for their treatment. Spinal imaging with plain and gadolinium enhanced MRI helps in identifying the hematoma, differentiating it from other pathologies and also exactly delineates the extent of hematoma in sagittal, coronal and axial plains. Coagulopathy should be reversed immediately and should be followed by surgical evacuation of hematoma. The neurological outcome reported in the literature is bad, with only a few patients showing mild improvement. ${ }^{3}$ But our patient has shown dramatic improvement with early reversal of coagulopathy and surgical evacuation. Hence, a high index of suspicion is required in patients on anticoagulation presenting with rapid onset paraparesis/ quadriparesis. Timely identification and early surgical intervation may result in a good neurological recovery.

\section{Conclusion}

Anticoagulants can rarely cause conus hematomyelia. Early reversal of anticoagulation and surgical evacuation of hematoma can lead to good neurological recovery.

\section{Bibliography}

[1]. Cakirer S, Basak M, Galip GM: Cervical hematomyelia secondary to oral anticoagulant therapy: case report. Neuroradiology 43:1087-8, 2001

[2]. Domenicucci M, Ramieri A, Ciappetta P, Delfini R: Nontraumatic acute spinal subdural hematoma: report of five cases and review of the literature. J Neurosurg 91:65-73, 1999

[3]. Famularo G, Sajeva MR, Gasbarrone L: Warfarin-associated hematomyelia. Intern Med 53:623-6, 2014

[4]. Kreppel D, Antoniadis G, Seeling W: Spinal hematoma: a literature survey with meta-analysis of 613 patients. Neurosurg Rev 26: $1-49,2003$

[5]. Leep Hunderfund AN, Wijdicks EFM: Intramedullary spinal cord hemorrhage (hematomyelia). Rev Neurol Dis 6:E54-61, 2009

[6]. Morandi X, Riffaud L, Chabert E, Brassier G: Acute nontraumatic spinal subdural hematomas in three patients. Spine (Phila Pa 1976) 26:E547-51, 2001

[7]. Pisani R, Carta F, Guiducci G, Silvestro C, Davini MD: Hematomyelia during anticoagulant therapy. Surg Neurol 24:578-80, 1985

[8]. Pullarkat VA, Kalapura T, Pincus M, Baskharoun R: Intraspinal hemorrhage complicating oral anticoagulant therapy: an unu sual case of cervical hematomyelia and a review of the literature. Arch Intern Med 160:237-40, 2000

\section{Figure Legends}

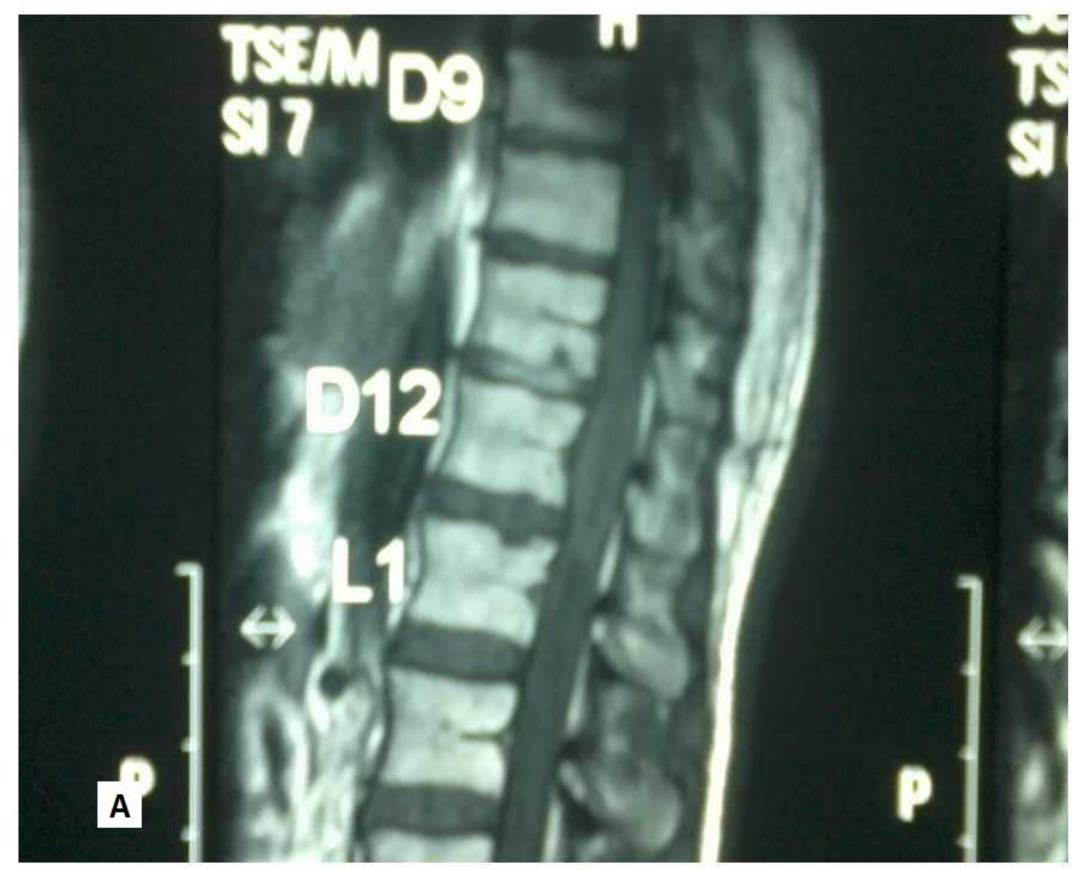



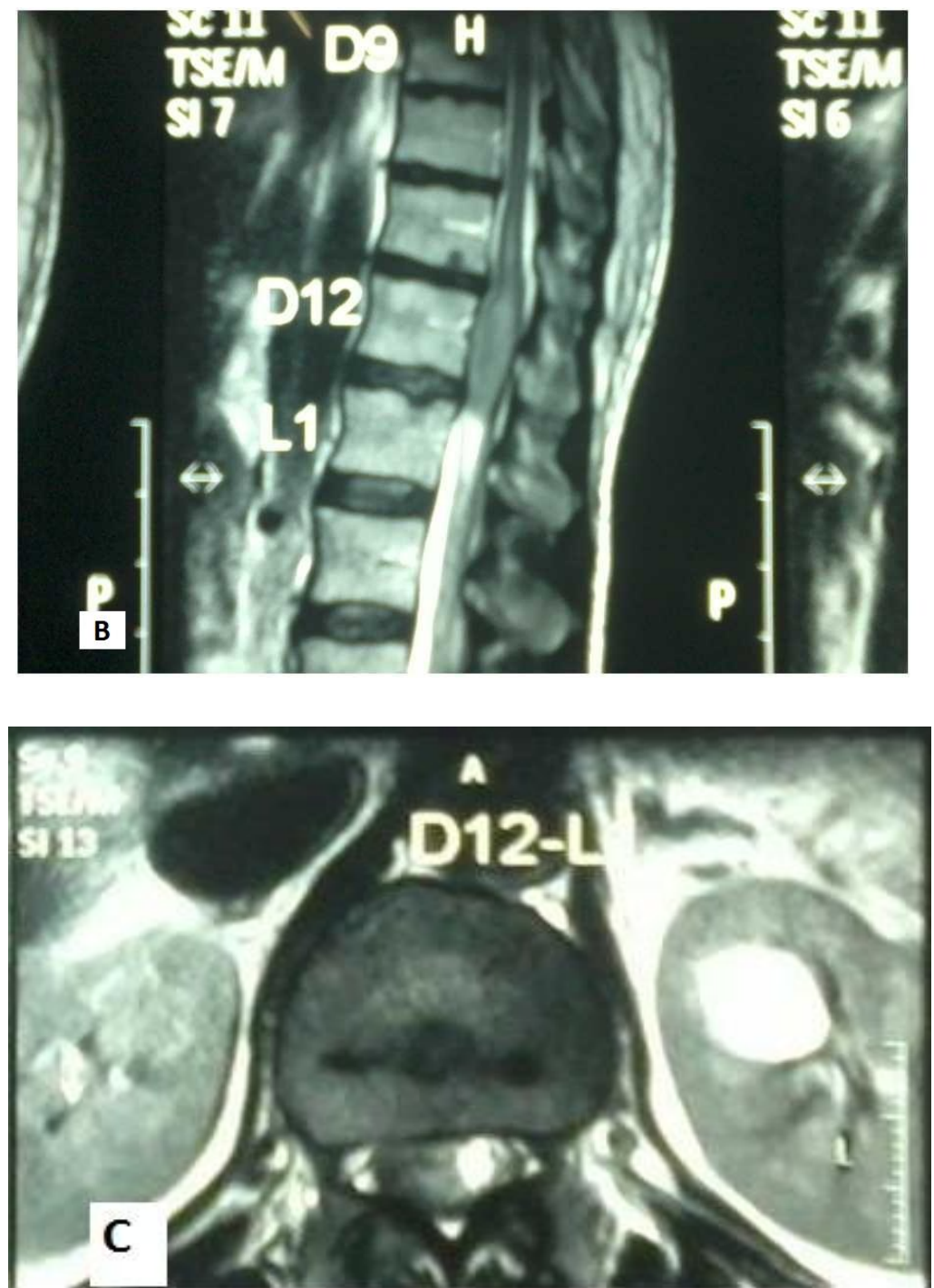

Fig 1: Sagittal T1W (A) and T2W (B) MR images of dorsolumbar spine showing the bleed in conus and extending downwards. The bleed is isointense to hypointense to the cord parenchyma in T1W images and is hyperintense in T2W images. Cord oedema is seen extending upwards up to D-10 level. Axial T2 W (C) MR image of conus showing hyperintense intramedullary bleed .

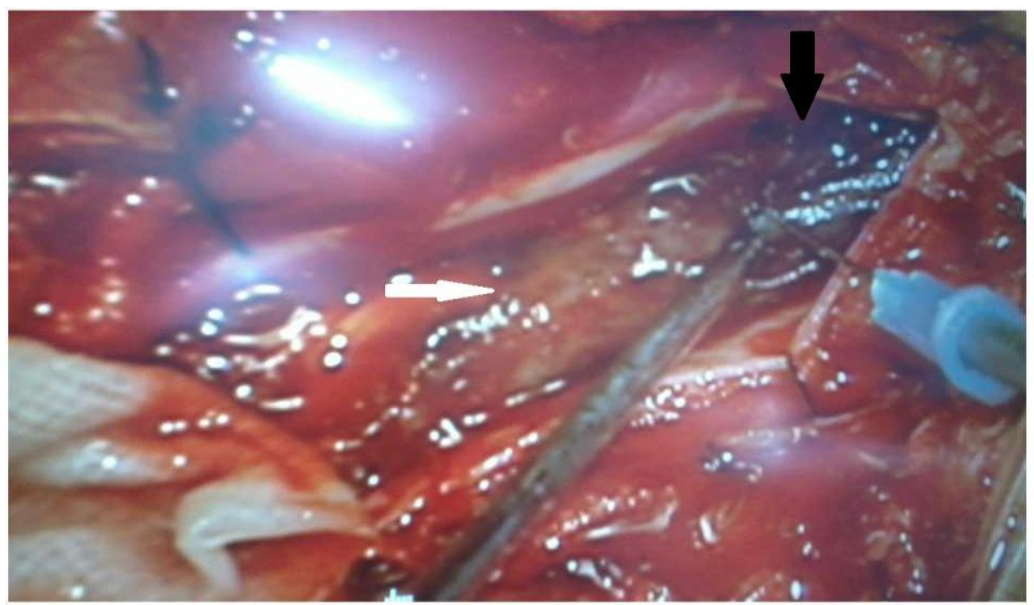

Fig 2: Intraoperative picture after opening the duramater and focally incising the arachnoid mater at the level of conus. The subarachnoid space was filled with blood (white arrow ) and the conus was discoloured brownishred due to hematoma (black arrow). A midline myelotomy revealed a dark liquefied haematoma. 